\title{
Three-dimensional speckle tracking echocardiography-derived left atrial deformation analysis in acromegaly (Results from the MAGYAR-Path Study)
}

\author{
Árpád Kormányos $\mathrm{MD}^{1}$ | Péter Domsik $\mathrm{MD}, \mathrm{PhD}^{1}$ | Anita Kalapos $\mathrm{MD}, \mathrm{PhD}^{1}$ | \\ Zsuzsanna Valkusz MD, $\mathrm{PhD}^{2}$ | Csaba Lengyel MD, PhD ${ }^{2}$ | Tamás Forster MD, PhD, DSc, \\ FESC, FACC ${ }^{1}$ | Attila Nemes MD, PhD, DSc, FESC ${ }^{1}$
}

\author{
${ }^{1}$ 2nd Department of Medicine and \\ Cardiology Centre, Medical Faculty, Albert \\ Szent-Györgyi Clinical Center, University of \\ Szeged, Szeged, Hungary \\ ${ }^{2} 1$ st Department of Medicine, Medical \\ Faculty, Albert Szent-Györgyi Clinical \\ Center, University of Szeged, Szeged, \\ Hungary \\ Correspondence \\ Attila Nemes, 2nd Department of Medicine \\ and Cardiology Center, Medical Faculty, \\ Albert Szent-Györgyi Clinical Center, \\ University of Szeged, Szeged, Hungary. \\ Email: nemes.attila@med.u-szeged.hu
}

Introduction: Acromegaly is a chronic and disfiguring hormonal disease caused by a benign growth hormone-secreting pituitary adenoma in most of the cases. The objective of this study was to investigate three-dimensional (3D) speckle tracking echocardiography (3DSTE)-derived left atrial (LA) volumetric and functional properties in patients with acromegaly. It was also examined whether the activity of acromegaly is associated with further alterations in these LA parameters.

Methods: A total of 23 patients with acromegaly were involved in this study. Due to inadequate image quality, 4 patients were excluded from the 3DSTE analysis. The mean age of the remaining acromegalic subjects was $58.5 \pm 13.1$ years ( 7 males). The control group consisted of 21 age- and gender-matched healthy subjects (mean age $51.8 \pm 10.3$ years, 10 males).

Results: Maximum $(44.8 \pm 12.7 \mathrm{~mL} \quad$ vs $78.7 \pm 25.4 \mathrm{~mL}, \quad P<.05)$, minimum $(24.1 \pm 9.0 \mathrm{~mL}$ vs $44.5 \pm 15.5 \mathrm{~mL}, P<.05)$, and preatrial contraction $(35.5 \pm 11.2 \mathrm{~mL}$ vs $66.0 \pm 22.8 \mathrm{~mL}, P<.05)$ LA volumes were significantly different between all acromegaly patients compared with controls. Increased peak global and mean segmental radial and 3D strains and decreased LA circumferential strain (CS) could be detected in all acromegaly patients as compared to healthy subjects. Significant correlation could be detected between insulin-like growth factor 1 (IGF-1) index and peak superior LA-CS $(r=.49, P=.05)$ in acromegaly.

Conclusion: Acromegaly is associated with increased LA volumes and changes in LA functional properties.

KEYWORDS

cardiomyopathy, hormone structure/function, left atrium, strain, three-dimensional echocardiography

\section{1 | INTRODUCTION}

Acromegaly is a chronic and disfiguring hormonal disease caused by a benign growth hormone $(\mathrm{GH})$ secreting pituitary adenoma in most of the cases. ${ }^{1}$ Cardiovascular complications are associated with the long-term presence of GH and insulin-like growth factor I (IGF-I) and are the most common causes of morbidity and mortality in acromegaly. ${ }^{2}$ Hypertension, left ventricular hypertrophy (LVH), and consequent heart failure are among the most common cardiovascular complications of acromegaly, but specific arrhythmias and mitral valve regurgitation can also occur. ${ }^{3-5}$ 
Three-dimensional (3D) speckle tracking echocardiography (3DSTE) offers clinicians a new noninvasive tool in characterizing and understanding preclinical cardiac involvement via 3D strain analysis, which is unique to this tool. ${ }^{6}$ 3DSTE is a validated method for left atrial (LA) quantification as compared to two-dimensional (2D) echocardiography, 2DSTE, and volumetric real-time 3D echocardiography (RT3DE). ${ }^{6-8}$ The number of studies concerning acromegaly-associated LA volumetric and functional changes is limited. ${ }^{9}$ The objective of this study was to investigate 3DSTE-derived LA volumetric and functional properties in patients with acromegaly. It was also examined whether the activity of acromegaly is associated with further alterations in these LA parameters.

\section{METHODS}

\section{1 | Patient population}

A total of 23 patients with acromegaly were involved in this study. Due to inadequate image quality, 4 patients were excluded from the 3DSTE analysis. The mean age of the remaining acromegalic subjects was $58.5 \pm 13.1$ years, from which 7 subjects were male. Acromegaly was diagnosed according to relevant clinical standards: typical clinical features and elevated serum GH and/or IGF-1 levels not suppressible with oral glucose tolerant test (OGTT, $75 \mathrm{~g}$ ). ${ }^{5}$ The acromegaly patient group was further divided into two subgroups based on the activity of the disease. Patients were allocated to the active subgroup if serum $\mathrm{GH}$ levels

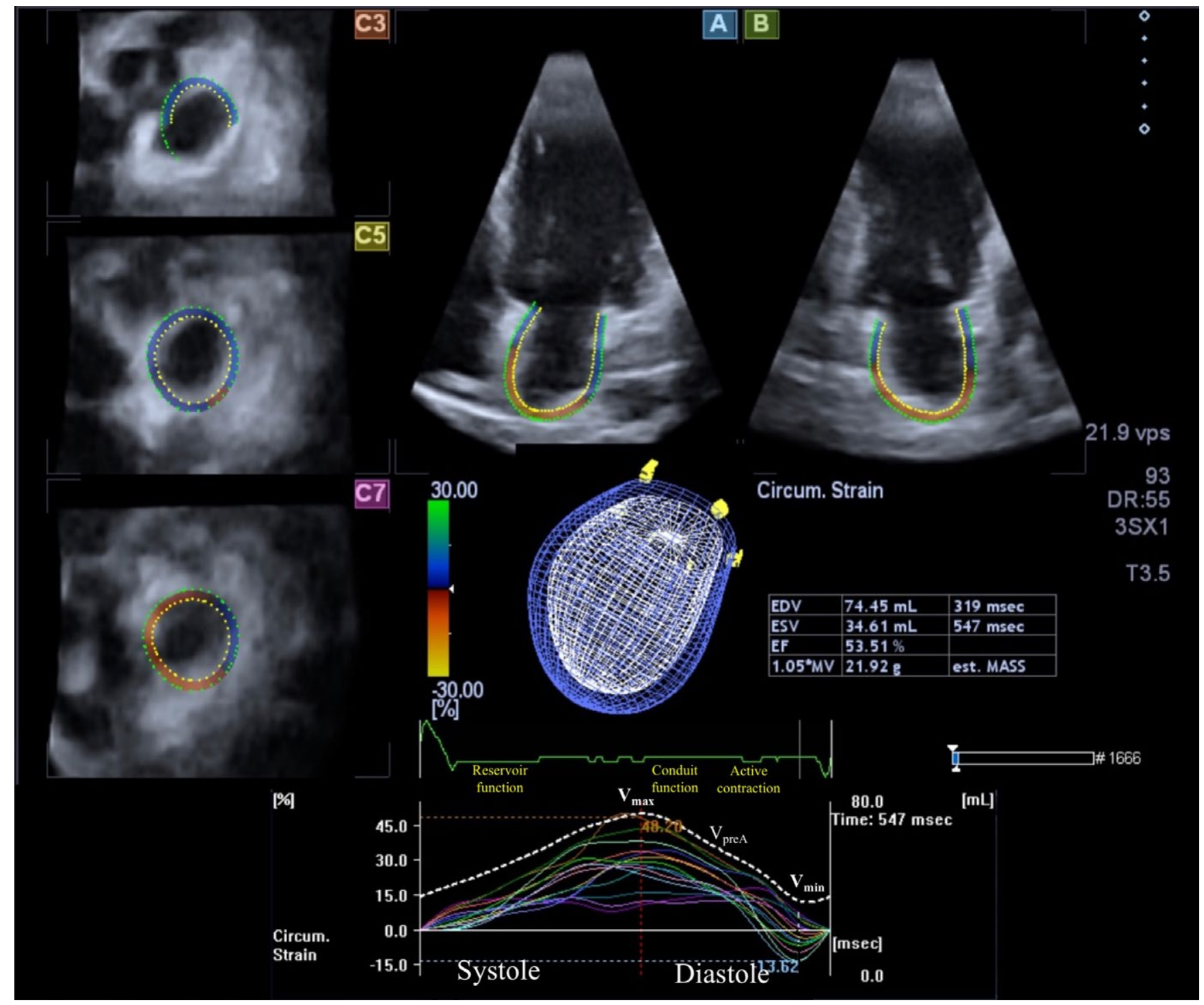

FIGURE 1 Three-dimensional (3D) speckle tracking echocardiographic assessment of the left atrium (LA) is displayed in an acromegaly patient. Apical four-chamber view (A), apical two-chamber view (B), short-axis view at basal (C3), midatrial (C5), and superior left atrial level (C7) are presented together with a 3D model of the LA and calculated volumetric data. Time-LA global volume (dashed line) and Time-LA circumferential segmental strain curves (colorized lines). EDV = end-diastolic volume; ESV = end-systolic volume; $E F=$ ejection fraction; $\mathrm{LA}=$ left atrium 
were above the standard threshold. ${ }^{5,10}$ Control group consisted of 21 age- and gender-matched healthy subjects (mean age $51.8 \pm 10.3$ years, 10 male). All data of acromegalic patients were taken from the MAGYAR-Path Study (Motion Analysis of the heart and Great vessels bY three-dimensionAl speckle-tRacking echocardiography in Pathological cases), which was created to assess diagnostic and prognostic value of 3DSTE-derived parameters ("magyar" means "Hungarian" in Hungarian language). The study complied with the Declaration of Helsinki and was approved by the institutional human research committee. All patients and control subjects gave informed consent.

\subsection{Two-dimensional Doppler and tissue Doppler echocardiography}

All acromegalic patients and negative control subjects underwent a complete two-dimensional (2D) transthoracic echocardiographic assessment using a Toshiba Artida imaging system (Toshiba Medical Systems, Tokyo, Japan) with a PST-30SBP $(1-5 \mathrm{MHz})$ phased array transducer. According to routine clinical standards, complete 2D Doppler study was performed in all cases including quantification of LV dimensions, volumes, ejection fraction, and LA dimensions. $^{11}$

\section{3 | Three-dimensional speckle tracking echocardiography}

Three-dimensional speckle tracking echocardiography measurements were performed using the same Toshiba Artida echocardiography equipment with a PST-25SX matrix-array transducer with 3DSTE capability. Full-volume 3D datasets were obtained by combining $6 \mathrm{R}$-wave triggered wedge-shaped subvolumes during 6 cardiac cycles. Subvolumes were registered during a single breath-hold and constant $R R$ interval from the apical window. Sector widths of the subvolumes were chosen to be as narrow as possible thus improving spatial resolution and providing improved endocardial border detection. Registered datasets were analyzed off line using 3D Wall Motion Tracking software version 2.7 (Toshiba Medical Systems). The software chose an apical four-chamber (AP4CH) view and an apical two-chamber (AP2CH) view together with 3 short-axis
TABLE 1 Demographic, clinical, and two-dimensional echocardiographic data of patients with acromegaly and controls

\begin{tabular}{|c|c|c|c|}
\hline & Controls $(n=21)$ & $\begin{array}{l}\text { Acromegaly patients } \\
(n=19)\end{array}$ & $P$-value \\
\hline \multicolumn{4}{|l|}{ Risk factors } \\
\hline Age (years) & $51.8 \pm 10.3$ & $58.5 \pm 13.1$ & .08 \\
\hline Male gender (\%) & $10(48)$ & $7(37)$ & .54 \\
\hline $\mathrm{BSA}\left(\mathrm{m}^{2}\right)$ & $2.0 \pm 0.2$ & $2.1 \pm 0.3$ & .26 \\
\hline Hypertension (\%) & $0(0)$ & $11(58)$ & $<.0001$ \\
\hline Hypercholesterolemia (\%) & $0(0)$ & $8(42)$ & .001 \\
\hline Diabetes mellitus (\%) & $0(0)$ & $3(16)$ & .1 \\
\hline \multicolumn{4}{|l|}{ Medications } \\
\hline$\beta$-blockers (\%) & $0(0)$ & $5(26)$ & .02 \\
\hline ACE inhibitors (\%) & $0(0)$ & $8(42)$ & .001 \\
\hline ARB (\%) & $0(0)$ & $5(30)$ & .02 \\
\hline Diuretics (\%) & $0(0)$ & $6(32)$ & .007 \\
\hline \multicolumn{4}{|l|}{ Two-dimensional echocardiography } \\
\hline LA diameter (mm) & $33.9 \pm 3.4$ & $42.1 \pm 5.6$ & $<.0001$ \\
\hline LA diameter/BSA $\left(\mathrm{mm} / \mathrm{m}^{2}\right)$ & $17.8 \pm 2.3$ & $20.8 \pm 3.3$ & .005 \\
\hline LA volume/BSA $\left(\mathrm{mL} / \mathrm{m}^{2}\right)$ & $25.1 \pm 8.3$ & $31.5 \pm 13.9$ & .08 \\
\hline LV end-diastolic diameter ( $\mathrm{mm}$ ) & $47.4 \pm 7.3$ & $51.1 \pm 5.4$ & .07 \\
\hline LV end-diastolic volume (mL) & $107.6 \pm 38.6$ & $128.3 \pm 28.6$ & .06 \\
\hline LV end-systolic diameter (mm) & $30.3 \pm 4.8$ & $32.0 \pm 5.0$ & .27 \\
\hline LV end-systolic volume (mL) & $36.3 \pm 13.0$ & $42.7 \pm 15.7$ & .17 \\
\hline Interventricular septum (mm) & $9.2 \pm 1.7$ & $10.1 \pm 1.5$ & .07 \\
\hline LV posterior wall (mm) & $9.4 \pm 1.9$ & $10.9 \pm 1.9$ & .02 \\
\hline LV ejection fraction (\%) & $65.4 \pm 6.3$ & $66.7 \pm 7.7$ & .56 \\
\hline E velocity $(\mathrm{cm} / \mathrm{s})$ & $66.5 \pm 14.1$ & $62.3 \pm 10.4$ & .30 \\
\hline A velocity $(\mathrm{cm} / \mathrm{s})$ & $65.5 \pm 15.9$ & $79.3 \pm 15.7$ & .01 \\
\hline E/A ratio & $1.1 \pm 0.3$ & $0.8 \pm 0.2$ & .002 \\
\hline
\end{tabular}

$\mathrm{ARB}=$ angiotensine $\quad$ II receptor blocker; $\mathrm{ACE}=$ angiotensine-converting enzyme inhibitor; $\mathrm{BSA}$ = body surface area; $\mathrm{LA}=$ left atrium; $\mathrm{LV}=$ left ventricle. 
views of the LA at end-diastole automatically. After image optimization, the reader detected the endocardial border by placing 6-8 markers to the edge of the septum-mitral annulus (MA) toward the edge of the lateral wall-MA (Figures 1 and 2). Then, the software automatically reconstructed the endocardial surface of the LA and performed volumetric and strain analyses. The frame rate changed between 27 and 30/s, and volume rates were between 27 and 30 vps during 3DSTE assessments.

\section{4 | 3DSTE-derived LA volumetric measurements}

The following volumetric data were obtained from the 3DSTE datasets (Figure 1):

- End-systolic maximum LA volume $\left(\mathrm{V}_{\max }\right.$, before mitral valve opening).

- Early diastolic LA volume before atrial contraction $\left(\mathrm{V}_{\mathrm{PreA}}\right.$, at the time of the P-wave on the ECG).

- End-diastolic minimum LA volume $\left(\mathrm{V}_{\min }\right.$, before mitral valve closure).

Based on the acquired volumetric data, several functional parameters were calculated representing systolic reservoir and diastolic conduit and active contraction LA functional phases. ${ }^{8}$

\subsection{1 | Reservoir function}

- Total stroke volume $(\mathrm{SV})=\mathrm{V}_{\max }-\mathrm{V}_{\text {min }}$

- Total emptying fraction (EF) = Total $S V / \mathrm{V}_{\max }$

\subsection{2 | Conduit function}

- Passive $\mathrm{SV}=\mathrm{V}_{\max }-\mathrm{V}_{\text {preA }}$

- Passive $E F=$ Passive $S V / V_{\max }$

\subsection{3 | Active contraction}

- Active $S V=V_{\text {preA }}-V_{\text {min }}$

- Active $\mathrm{EF}=$ Active $\mathrm{SV} / \mathrm{V}_{\text {preA }}$

\section{5 | 3DSTE-derived LA strain assessments}

Using the same 3D datasets, the software obtained several linear (radial [RS], longitudinal [LS], and circumferential [CS]) and two complex (3D [3DS] and area [AS]) LA strains automatically. Global, mean segmental, and regional strains were also calculated. Peak strains were measured during the LA reservoir phase

TAB LE 2 Comparison of 3DSTE-derived volumetric left atrial parameters between patients with acromegaly and controls

\begin{tabular}{|c|c|c|c|c|}
\hline & Controls $(n=21)$ & $\begin{array}{l}\text { Acromegaly patients } \\
(n=19)\end{array}$ & $\begin{array}{l}\text { Active acromegaly patients } \\
(\mathrm{n}=11)\end{array}$ & $\begin{array}{l}\text { Inactive acromegaly } \\
\text { patients }(n=8)\end{array}$ \\
\hline \multicolumn{5}{|l|}{ Calculated volumes } \\
\hline $\mathrm{V}_{\max } / \mathrm{BSA}\left(\mathrm{mL} / \mathrm{m}^{2}\right)$ & $23.5 \pm 7.7$ & $38.7 \pm 13.0^{*}$ & $37.8 \pm 15.7^{*}$ & $39.9 \pm 9.0^{*}$ \\
\hline $\mathrm{V}_{\min }(\mathrm{mL})$ & $24.1 \pm 9.0$ & $44.5 \pm 15.5^{*}$ & $44.4 \pm 16.5^{*}$ & $44.6 \pm 15.3^{*}$ \\
\hline $\mathrm{V}_{\mathrm{pre}} \mathrm{A}(\mathrm{mL})$ & $35.5 \pm 11.2$ & $66.0 \pm 22.8^{*}$ & $62.5 \pm 23.8^{*}$ & $70.7 \pm 21.9^{*}$ \\
\hline $\mathrm{V}_{\max } / \mathrm{BSA}\left(\mathrm{mL} / \mathrm{m}^{2}\right)$ & $18.8 \pm 6.8$ & $32.5 \pm 12.1^{*}$ & $31.8 \pm 14.7^{*}$ & $33.5 \pm 8.1^{*}$ \\
\hline \multicolumn{5}{|l|}{ Stroke volumes } \\
\hline TASV (mL) & $20.7 \pm 6.8$ & $34.2 \pm 12.8^{*}$ & $30.3 \pm 12.1^{*}$ & $39.6 \pm 12.5^{*}$ \\
\hline TASV/BSA $\left(\mathrm{mL} / \mathrm{m}^{2}\right)$ & $11.0 \pm 4.2$ & $16.6 \pm 5.7^{*}$ & $15.2 \pm 6.4$ & $18.6 \pm 4.1^{*}$ \\
\hline AASV/BSA $\left(\mathrm{mL} / \mathrm{m}^{2}\right)$ & $6.3 \pm 3.5$ & $10.5 \pm 4.5^{*}$ & $9.2 \pm 5.4$ & $12.3 \pm 2.3^{*}$ \\
\hline \multicolumn{5}{|l|}{ Emptying fractions } \\
\hline TAEF (\%) & $46.9 \pm 10.2$ & $43.2 \pm 8.7$ & $40.2 \pm 8.0$ & $47.3 \pm 8.5$ \\
\hline PAEF (\%) & $21.4 \pm 9.0$ & $16.3 \pm 7.7$ & $16.5 \pm 9.2$ & $16.1 \pm 5.6$ \\
\hline AAEF (\%) & $32.5 \pm 10.4$ & $31.9 \pm 9.4$ & $28.0 \pm 9.7^{* *}$ & $37.5 \pm 6.0$ \\
\hline
\end{tabular}

AASV = active atrial stroke volume; $\mathrm{AAEF}=$ active atrial emptying fraction; $\mathrm{BSA}=$ body surface area; PAEF = passive atrial emptying fraction; $\mathrm{PASV}=$ passive atrial stroke volume; TAEF = total atrial emptying fraction; TASV = total atrial stroke volume; $\mathrm{V}_{\max }=$ maximum left atrial volume; $\mathrm{V}_{\min }=$ minimum left atrial volume; $\mathrm{V}_{\text {preA }}=$ left atrial volume before atrial contraction .

${ }^{*} P<.05$ vs controls.

${ }^{* *} P<.05$ vs inactive acromegaly. 
in systole, while strains at atrial contraction were obtained at end-diastole (LA systole) to characterize LA booster pump function (Figure 1). ${ }^{7}$

\subsection{Statistical analysis}

All data are reported as mean \pm standard deviation. $P$ values $<.05$ were considered significant. For categorical variables, Fisher's exact test was used. Shapiro-Wilks test was used to test normal distribution in every dataset. Homogeneity of variance was assessed using Levene's test for equality of variances. Student's $t$ test was used for datasets following normal distribution and Mann-Whitney-Wilcoxon test was used for datasets that were not normally distributed. Correlations were assessed by calculating Pearson's correlation coefficients. RStudio was used for statistical analysis (RStudio Team, RStudio: Integrated Development for R. RStudio, Inc., Boston, MA, 2015). For offline data analysis and graph creation, a commercial software package was used (MATLAB 8.6, The MathWorks Inc., Natick, MA, 2015).

\section{3 | RESULTS}

\subsection{Demographic data of patients with acromegaly}

Demographic and clinical data are presented in Table 1. Across all acromegalic patients, the mean level of serum $\mathrm{GH}$ was $5.73 \pm 6.89 \mathrm{ng} / \mathrm{mL}$ and mean IGF-1 serum concentration was $332 \pm 210 \mathrm{ng} / \mathrm{mL}$. Patients who were prior hypophysectomy or who underwent the surgery but had hormonally active remnant tissue or medically managed patients having elevated IGF-1 level despite long-acting somatostatine analogue therapy were placed in the active acromegalic subgroup $(n=11)$. In this group, the mean level of IGF-1 was $418 \pm 207 \mathrm{ng} / \mathrm{mL}$, and mean GH level was $5.52 \pm 3.94 \mathrm{ng} / \mathrm{mL}$. Patients who had normal IGF-1 and/or normal GH serum levels or normal GH nadir levels after OGTT during pegvisomant, bromocriptine, or long-acting somatostatin analogue therapy or patients who underwent successful hypophysectomy made up the inactive acromegalic subgroup $(n=8)$. In this group, the mean level of IGF-1 was $176 \pm 102 \mathrm{ng} / \mathrm{mL}$, mean GH level was $6.11 \pm 10.98 \mathrm{ng} / \mathrm{mL}$, and mean nadir hGH level after OGTT was $2.08 \pm 2.06 \mathrm{ng} / \mathrm{mL}$.

TAB LE 3 Comparison of 3DSTE-derived regional peak left atrial strain parameters between patients with acromegaly and controls

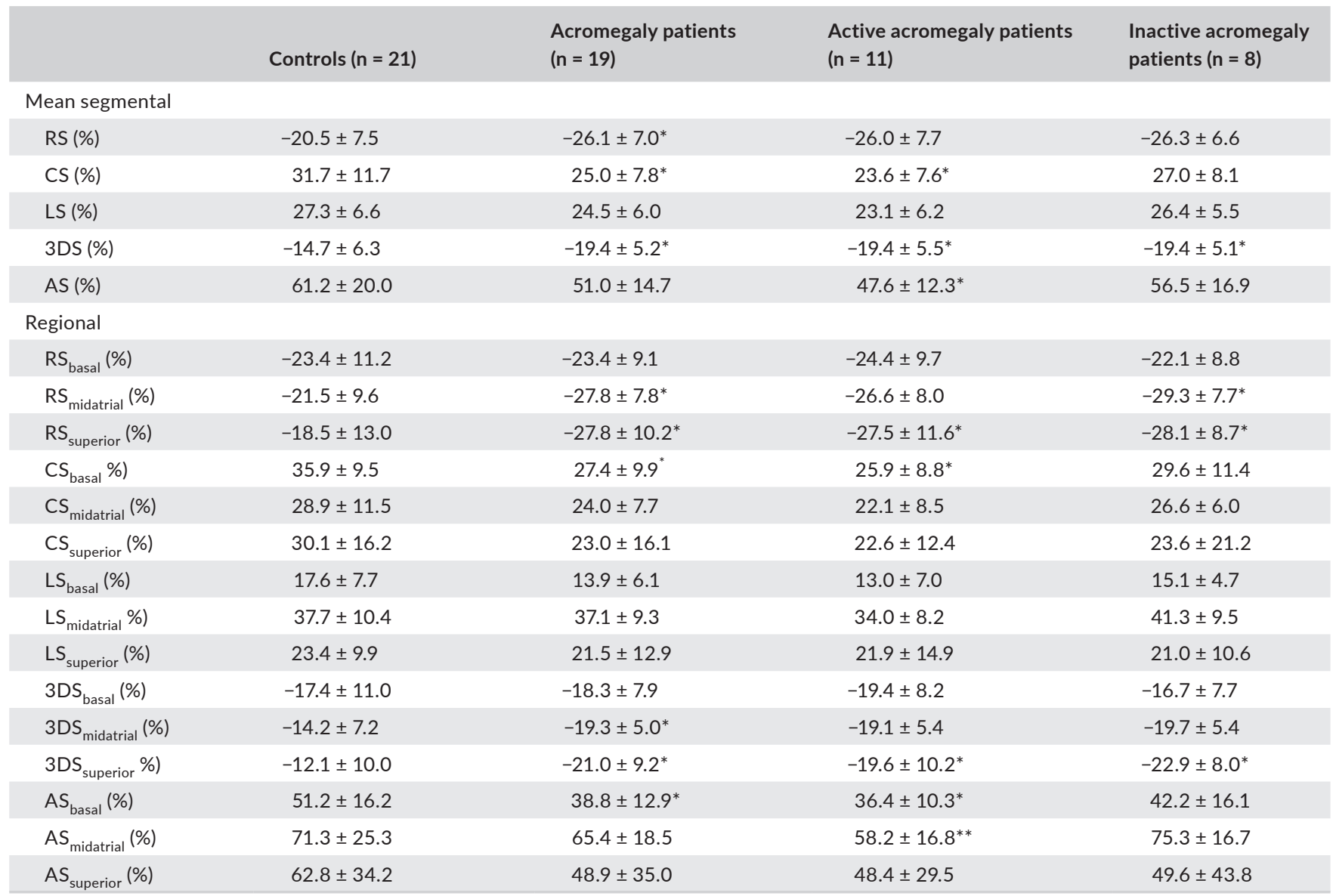

3DS = three-dimensional strain; $A S=$ area strain; $C S$ = circumferential strain; $L S=$ longitudinal strain; RS = radial strain.

${ }^{*} P<.05$ vs controls.

${ }^{* *} P<.05$ vs inactive acromegaly. 


\section{2 | Two-dimensional echocardiographic data}

Out of 2D echocardiography parameters, LA diameter, body surface area (BSA)-indexed LA diameter, and LV posterior wall thickness were significantly different between the patient populations $(P<.0001, P=.005, P=.02$, respectively). Early filling transmitral flow velocity $(E)$ was not significantly different; however, late filling transmitral flow velocity $(A)$ and $E / A$ ratio differed significantly between controls and acromegaly patients $(P=.01$ and $P=.002$, respectively). Among all acromegaly patients, mild-to-moderate mitral regurgitation was present in 7 cases (Table 1).

\section{3 | 3DSTE-derived volumes and volume-based LA functional properties}

All three LA volumetric parameters $\left(\mathrm{V}_{\max }, \mathrm{V}_{\text {preA }}, \mathrm{V}_{\min }\right)$ were significantly different between all acromegaly patients $(P \leq .0001$, $P \leq .0001, P \leq .0001$, respectively), active $(P=.004, P=.004$, $P \leq .0001$, respectively), and inactive acromegaly patients ( $P \leq .0001, P \leq .0001, P=.0001$, respectively) compared with negative controls. Similarly, all BSA-indexed $V_{\max }, V_{\text {preA }}$, and $V_{\text {min }}$ were significantly different between all acromegaly patients $(P=.0003$, $P=.0003, P=.0004$, respectively), active $(P=.03, P=.008$, $P=.0004$, respectively), and inactive acromegaly patients ( $P=.0003, P=.002, P=.0006$, respectively). Out of LA volumebased functional properties, only total and active SVs differed significantly between all acromegaly patients and healthy controls ( $P=.0003, P=.0002$, respectively). BSA-indexed total and active
SVs showed similar differences $(P=.003, P=.006$, respectively). Only total SV was significantly increased in active acromegaly patients as compared to that of the control group $(P=.03)$. Significant differences could be demonstrated between inactive acromegaly patients and control patients regarding all three stroke volumes (total, passive, and active SVs) $(P<.0001, P=.03, P<.0001$, respectively). From BSA-indexed parameters, total and active SVs differed significantly $(P=.0006, P=.0004$, respectively). Between the active and inactive acromegaly patient subgroups, only active EF differed significantly $(P=.03)$ (Table 2$)$. There was no significant correlation between volumetric parameters and mean $\mathrm{GH}$, IGF-1, or IGF-1 index.

\section{4 | 3DSTE-derived peak LA strain parameters}

Increased global and mean segmental peak RS ( $P=.04, P=.02)$ and 3DS $(P=.02, P=.02)$ and decreased global and mean segmental CS $(P=.02, P=.04)$ could be detected in all acromegaly patient groups as compared to the group of healthy subjects (Table 3, Figure 3). Global and mean segmental peak CS $(P=.02, P=.05)$ and mean segmental AS $(P=.05)$ were found to be diminished, while mean segmental 3DS were increased $(P=.05)$ in active acromegaly patients as compared to controls. Significantly increased mean segmental 3DS were found in data of the inactive acromegaly subgroup compared with healthy subjects $(P=.03$ ) (Table 3 ). Regional differences in all peak strains between all acromegaly patients, active and inactive acromegaly patients, and controls are demonstrated in Table 3. Significant correlation could be detected in the acromegaly group

\section{Control patient}

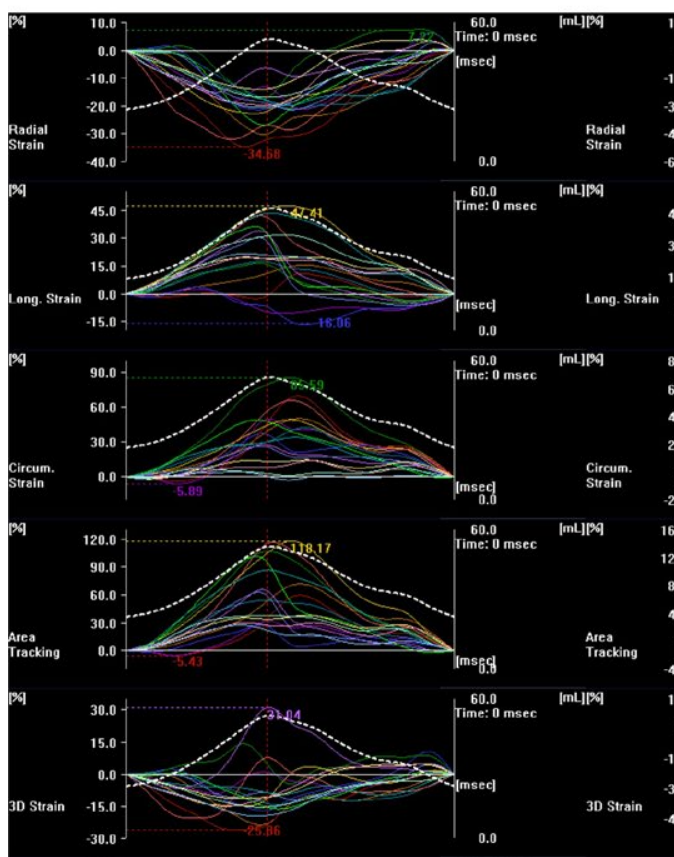

\section{Active acromegaly patient}

\section{Inactive acromegaly patient}

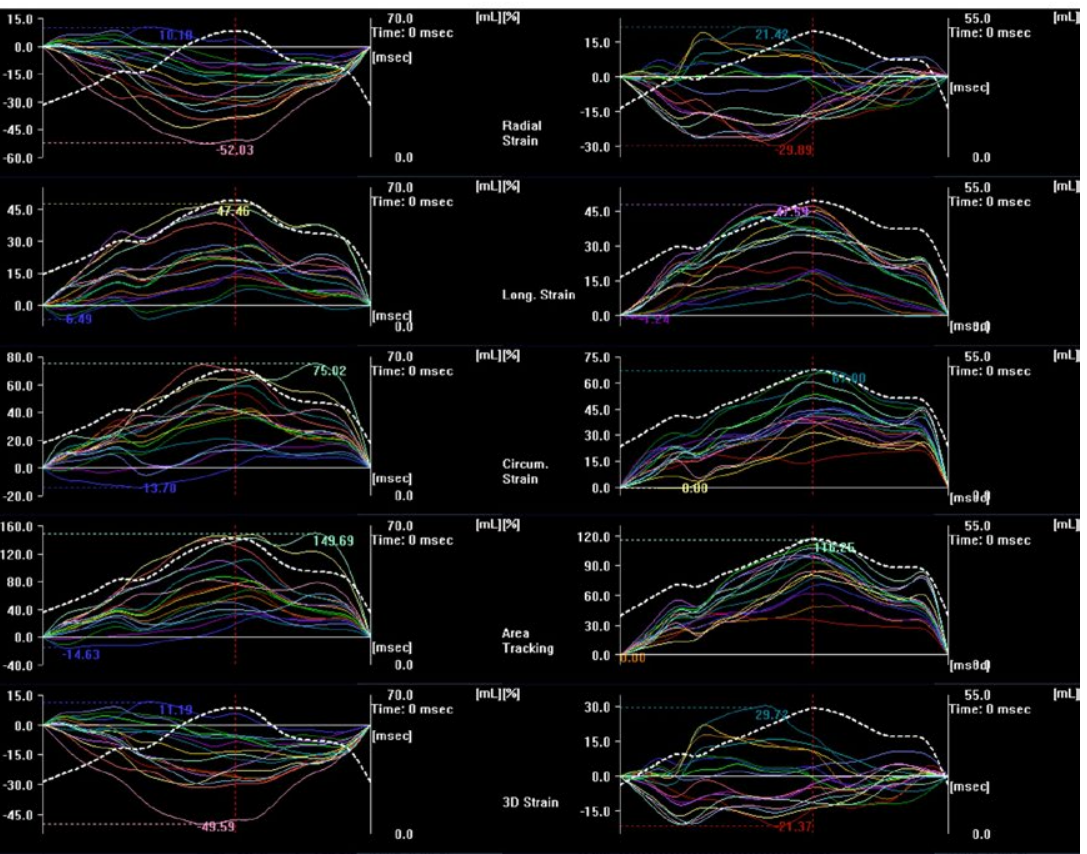

FIGURE 2 Three-dimensional (3D) speckle tracking echocardiographic assessment of all types of (segmental) strains (colored lines) and left atrial volume change (dashed white line) over cardiac cycle in a control subject and in active and inactive acromegalics 
between IGF-1 index and superior peak CS $(r=.49, P=.05)$. In the active acromegaly subgroup, significant correlations were found between the basal peak CS and serum IGF-1 and IGF-1 index levels $(r=.76, P=.007 ; r=.69, P=.02)$ (Figure 4).

\section{5 | 3DSTE-derived LA strains at atrial contraction}

Increased global and mean segmental 3DS $(P=.02, P=.02$, respectively) and mean segmental RS and LS $(P=.03, P=.02$, respectively) could be detected in all acromegaly patients compared with controls. While inactive acromegaly patients had the same global and mean segmental LA strain parameters as compared to that of controls, activity of acromegaly was associated with increased global and mean segmental RS $(P=.01, P=.007)$ and 3DS $(P=.02$, $P=.03)$. Moreover, significant differences could be detected between global and mean segmental RS $(P=.01, P=.03)$ and mean segmental 3DS $(P=.05)$ of active and inactive acromegaly patients (Table 4, Figure 3). Regional differences in all strains at atrial contraction between all acromegaly patients, active and inactive acromegaly patients, and controls are demonstrated in Table 4.

\section{4 | DISCUSSION}

Acromegaly is a rare, chronic, progressive, and insidious disorder characterized by excess secretion of $\mathrm{GH}$ and elevated circulating levels of IGF-I, generally caused by a pituitary adenoma. ${ }^{12}$ Acromegaly patients often have established systemic complications at diagnosis with increased morbidity and mortality mostly due to cardiovascular reasons. ${ }^{13}$ The pathophysiology underlying cardiac involvement in acromegaly is not clearly understood, elevated GH serum levels and consequent plasma volume expansion and direct anti-natriuretic effect, increased peripheral vascular resistance may be the cause. LVH and myocardial fibrosis are considered common findings in acromegalic cardiomyopathy in echocardiography studies. ${ }^{14}$ However, there is limited information on LA function in acromegaly. ${ }^{9}$

Newer echocardiographic modalities including 3D and/or STE enable detailed assessment of not only the LV, but atrial functions as well. ${ }^{8}$ 3DSTE uses a "block-matching" by strain analysis algorithm to accurately assess volumetric and functional properties of the LA during different cardiac cycles. ${ }^{8}$ Volumetric RT3DE is widely regarded as a highly accurate noninvasive method for volumetric

TAB LE 4 Comparison of 3DSTE-derived regional left atrial strain parameters at atrial contraction between patients with acromegaly and controls

\begin{tabular}{|c|c|c|c|c|}
\hline & Controls $(n=21)$ & $\begin{array}{l}\text { Acromegaly patients } \\
(n=19)\end{array}$ & $\begin{array}{l}\text { Active acromegaly patients } \\
(n=11)\end{array}$ & $\begin{array}{l}\text { Inactive acromegaly } \\
\text { patients }(n=8)\end{array}$ \\
\hline \multicolumn{5}{|l|}{ Mean segmental } \\
\hline CS (\%) & $15.0 \pm 8.6$ & $14.4 \pm 5.8$ & $12.7 \pm 6.0$ & $16.6 \pm 5.1$ \\
\hline LS (\%) & $9.3 \pm 4.3$ & $12.1 \pm 3.6^{*}$ & $12.2 \pm 3.8$ & $12.1 \pm 3.6$ \\
\hline AS (\%) & $21.6 \pm 11.7$ & $26.2 \pm 9.0$ & $23.6 \pm 8.7$ & $29.7 \pm 8.8$ \\
\hline \multicolumn{5}{|l|}{ Regional } \\
\hline $\mathrm{RS}_{\text {basal }}(\%)$ & $-10.7 \pm 7.4$ & $-12.9 \pm 6.4$ & $-14.1 \pm 7.4$ & $-11.2 \pm 4.7$ \\
\hline $\mathrm{RS}_{\text {midatrial }}(\%)$ & $-8.0 \pm 7.0$ & $-12.9 \pm 6.5^{*}$ & $-15.6 \pm 5.6^{*, * *}$ & $-9.0 \pm 6.0$ \\
\hline $\mathrm{RS}_{\text {superior }}(\%)$ & $-8.6 \pm 6.1$ & $-14.2 \pm 7.2^{*}$ & $-16.5 \pm 7.3^{*}$ & $-11.1 \pm 6.0$ \\
\hline $\mathrm{LS}_{\text {basal }}(\%)$ & $6.9 \pm 5.5$ & $6.7 \pm 4.2$ & $5.7 \pm 2.7$ & $8.1 \pm 5.5$ \\
\hline $\left.\mathrm{LS}_{\text {midatrial }} \%\right)$ & $11.4 \pm 6.7$ & $16.8 \pm 7.2^{*}$ & $17.7 \pm 5.7^{*}$ & $15.6 \pm 9.2$ \\
\hline $\mathrm{LS}_{\text {superior }}(\%)$ & $9.8 \pm 8.1$ & $13.2 \pm 10.5$ & $13.5 \pm 12.2$ & $12.8 \pm 8.4$ \\
\hline $3 \mathrm{DS}_{\text {basal }}(\%)$ & $-8.2 \pm 6.7$ & $-10.2 \pm 5.5$ & $-10.3 \pm 5.2$ & $-10.0 \pm 6.3$ \\
\hline $3 D S_{\text {midatrial }}(\%)$ & $-4.9 \pm 5.4$ & $-10.1 \pm 4.6^{*}$ & $-10.7 \pm 4.6^{*}$ & $-9.3 \pm 4.8^{*}$ \\
\hline $\left.3 \mathrm{DS}_{\text {superior }} \%\right)$ & $-6.8 \pm 5.3$ & $-10.7 \pm 6.4^{*}$ & $-11.8 \pm 7.1^{*}$ & $-9.3 \pm 5.4$ \\
\hline $\mathrm{AS}_{\text {basal }}(\%)$ & $20.7 \pm 9.2$ & $20.3 \pm 6.3$ & $17.8 \pm 6.2^{* *}$ & $23.8 \pm 4.9$ \\
\hline $\mathrm{AS}_{\text {midatrial }}(\%)$ & $22.6 \pm 12.8$ & $32.3 \pm 13.1^{*}$ & $30.9 \pm 12.7$ & $34.3 \pm 14.7^{*}$ \\
\hline
\end{tabular}

3DS = three-dimensional strain; AS = area strain; CS = circumferential strain; $L S=$ longitudinal strain; RS = radial strain.

${ }^{*} P<.05$ vs controls.

${ }^{* *} P<.05$ vs inactive acromegaly. 
measurements of LA due to its high spatial resolution. ${ }^{6}$ 3DSTE and RT3DE methodologies were found to be comparable, reproducible, and interchangeable. ${ }^{15}$ Also, 3DSTE has several advantages compared to 2DSTE. Mochizuki et al compared the two methods for LA chamber quantification, and they found that 3DSTE was similarly feasible as its 2D counterpart; however, the interobserver variability and time required for analysis were shorter in case of 3DSTE. Moreover, 2DSTE by its nature can only properly assess LA longitudinal function; however, 3DSTE is capable of measuring wall strains in circumferential direction and thus calculating area strain and 3D strain, as well. ${ }^{7}$ Furthermore, our group previously demonstrated the feasibility of 3DSTE in measuring LA volumes

\section{Peak global left atrial strains across various groups}

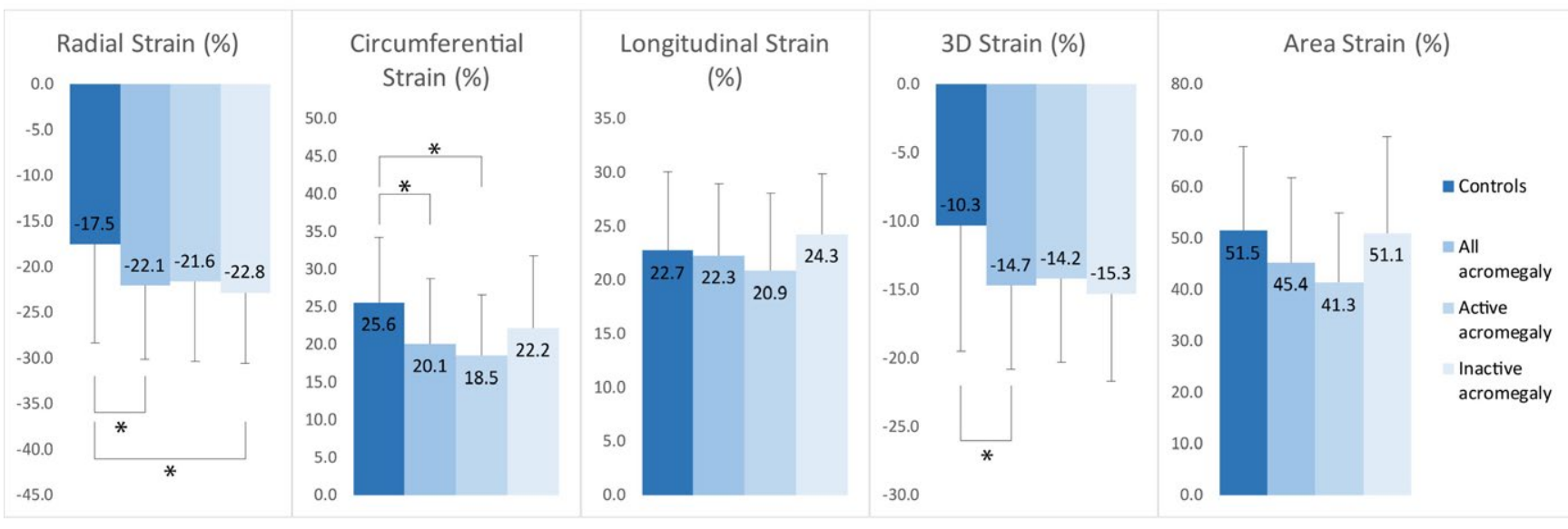

\section{Global left atrial strains at atrial contraction across various groups}

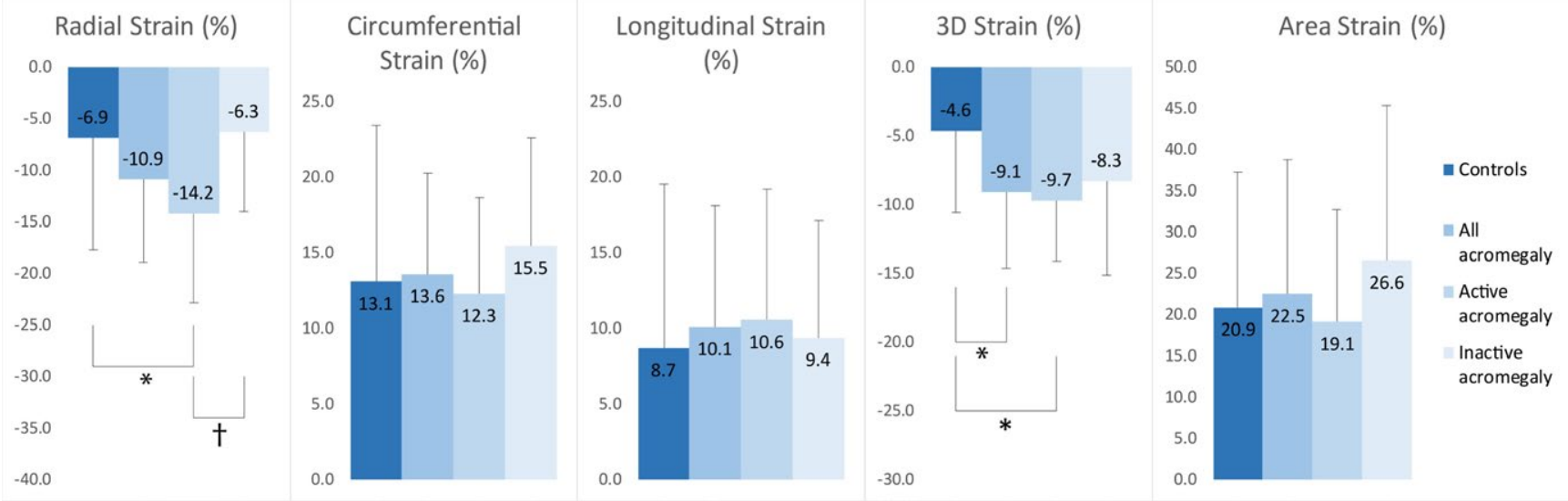

FIGURE 3 Bar charts showing global left atrial peak strains and strains at atrial contraction among the various groups. Numbers in the bars represent the mean strain values of each corresponding group. ${ }^{*} P<.05$ vs controls; $\uparrow P<.05$ vs inactive acromegaly

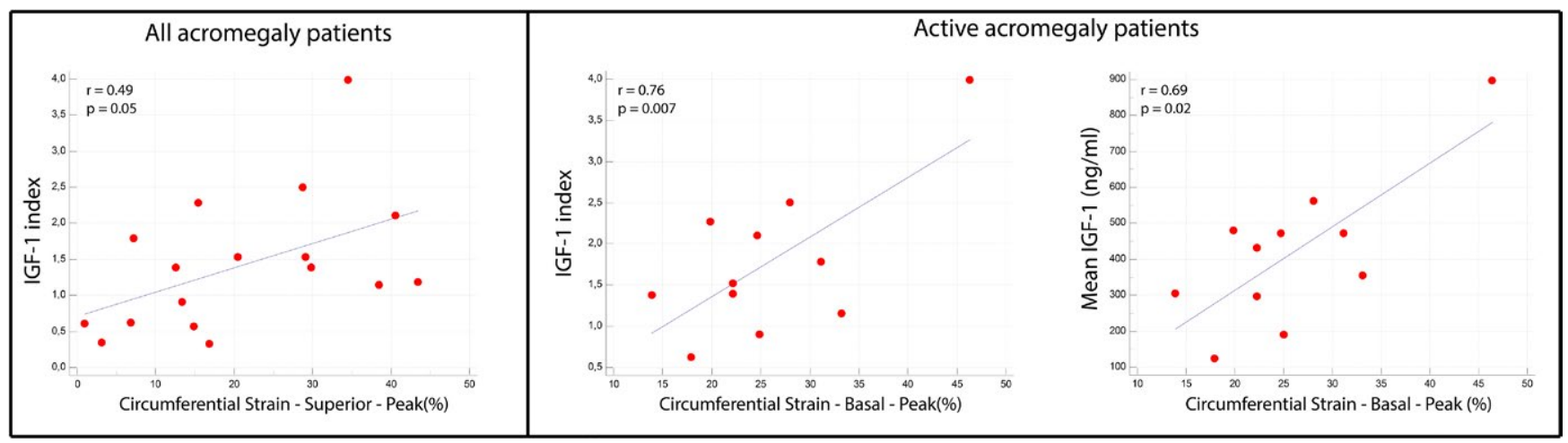

FIGURE 4 Significant correlations between IGF-1/IGF-1 index and segmental circumferential strain parameters in acromegalics 
and functional properties directly compared to the standard clinical 2D echocardiography technique. ${ }^{8}$

During the cardiac cycle, LA has a distinct phasic function. It serves as a reservoir during LV systole; during early LV diastole, it conducts blood from the pulmonary veins to the LV, and in late diastole, the LA augments LV filling through active contraction. ${ }^{16}$ Total, passive, and active SVs and EFs characterize the aforementioned LA functional properties, respectively. The complete 3D datasets enabled us to calculate global, mean segmental, and regional basal, midatrial and superior peak strains, and strains at atrial contraction as well for further characterization of the LA reservoir and atrial booster pump functions.

In a recent study, LA volumes (maximum, minimum, and presystolic) and LA mechanical functions were not significantly different in patients with active acromegaly compared with age- and gendermatched healthy controls. Additionally, serum levels of $\mathrm{GH}$ and IGF-1 did not correlate with LA mechanical functions. ${ }^{9}$ A previous animal study examined 11 cats with acromegaly; 6 of the subject animals had significantly enlarged LA. ${ }^{17}$ In a previous study presenting 3 acromegalic patients with heart failure, the LA was found to be enlarged in all three cases. ${ }^{18}$ To the best of the author's knowledge, there are no studies characterizing 3DSTE-derived LA volumes, volume-based functional properties, and strains in acromegaly patients. Significantly enlarged 3DSTE-derived LA volumes respecting cardiac cycle could be demonstrated in acromegalic patients as compared to controls regardless the activity of disease. Out of the functional properties, total and active atrial SVs were significantly different, but atrial EFs showed no difference across all acromegaly patients compared to healthy controls. The active acromegaly patients had enhanced atrial SVs on average compared to the healthy population, although the difference reached the level of significance only in case of the total atrial SV. The inactive acromegaly patient subgroup showed significantly augmented atrial SVs compared to the control group. Total and active atrial EFs were augmented in the inactive acromegaly population compared to the active acromegaly group, although only the difference of active atrial EF reached the level of significance. These results suggest that after proper treatment, preexisting LA volumetric changes do not recede, although enhanced LA SVs might suggest a compensatory mechanism. Strain analysis revealed decreased global peak CS and increased global peak RS and 3DS in acromegaly. In case of inactive acromegaly, the decreased peak CS receded and was not significantly different compared to this parameter of the healthy controls. Mean segmental peak LA strains showed similar results. In case of LA, CSs are thought to be the most sensitive parameter, and the above-described changes might suggest a somewhat reversible atrial dysfunction after proper treatment of acromegaly.

The above-described LA changes may be reactive to the LV changes that are characteristic in acromegaly. In the early stages, hyperkinetic syndrome and LVH are the primary changes (caused by increased Ca-sensitivity thus increased myocardial contractility); as the disease progresses, LVH becomes more prominent and diastolic ventricular filling becomes impaired. In the later stages, extracellular collagen deposition and thus reduced systolic and diastolic function, LV remodeling, and heart failure characterize the clinical picture. ${ }^{19-22}$ The remodeling of the LA might also occur, parallel to the $\mathrm{LVH}$, but the possible effect of collagen deposition cannot be ruled out either. The real mechanism behind the LA volumetric and functional alterations and LA remodeling is not known yet in acromegaly. The increased LA volumes and augmented SVs might be due to the decreased diastolic filling of the LV; however, the effect of hypertension and a certain degree of mitral regurgitation cannot be ruled out as these are common findings in acromegaly. Moreover, agerelated changes could have an effect, as well. The hormonal changes (GH and IGF-1) might also play a notable part in the remodelation of the LA.

\section{1 | Limitations}

The main limitation of this study is the low number of acromegaly patients involved. In the present study, we did not take into account for how long the acromegaly was active after the diagnosis, or for how long the disease was inactive in case of successful treatment. The possible effects of the specific drugs used in the treatment of acromegaly on LA volumetric, functional, and strain parameters were not explored in this study. In addition, the spatial resolution of $3 D S T E$ is relatively poor compared to that of $2 \mathrm{D}$ echocardiography. There are no reference values for 3DSTE-derived LA strain, volumetric and functional parameters at this time; therefore, LA data of control patients might differ compared to other studies. Due to the nature of the software used, only R-wave triggered measurements were possible; even though in case of LA, P-wave triggering is superior. ${ }^{23}$ The software is capable of measuring RS, but in case of the atria, it is thought to be a weak parameter due to the thinness of atrial walls. Even though 3DSTE is a feasible imaging modality for quantifying LA volumetric and strain assessments, further validation studies with larger populations are warranted.

\section{5 | CONCLUSION}

Acromegaly is associated with increased LA volumes and changes in LA functional properties.

\section{CONFLICT OF INTEREST}

None declared.

ORCID

Attila Nemes (iD http://orcid.org/0000-0002-7570-6214

\section{REFERENCES}

1. Sanno N, Teramoto A, Osamura RY, et al. Pathology of pituitary tumors. Neurosurg Clin N Am. 2003;14:25-39. 
2. Colao A, Ferone D, Marzullo P, et al. Systemic complications of acromegaly: epidemiology, pathogenesis, and management. Endocr Rev. 2004;25:102-152.

3. Clayton RN. Cardiovascular function in acromegaly. Endocr Rev. 2003;24:272-277.

4. Ramos-Levi AM, Marazuela M. Cardiovascular comorbidities in acromegaly: an update on their diagnosis and management. Endocrine. 2017;55:346-359.

5. Melmed S. Medical progress: acromegaly. $N$ Engl J Med. 2006;355:2558-2573.

6. Kleijn SA, Aly MF, Terwee CB, et al. Comparison between direct volumetric and speckle tracking methodologies for left ventricular and left atrial chamber quantification by three-dimensional echocardiography. Am J Cardiol. 2011;108:1038-1044.

7. Mochizuki A, Yuda S, Oi Y, et al. Assessment of left atrial deformation and synchrony by three-dimensional speckle-tracking echocardiography: comparative studies in healthy subjects and patients with atrial fibrillation. J Am Soc Echocardiogr. 2013;26:165-174.

8. Nemes A, Domsik P, Kalapos A, et al. Comparison of threedimensional speckle tracking echocardiography and twodimensional echocardiography for evaluation of left atrial size and function in healthy volunteers (results from the MAGYAR-Healthy Study). Echocardiography. 2014;31:865-871.

9. Ilter A, Kiris A, Kaplan S, et al. Atrial conduction times and left atrium mechanical functions in patients with active acromegaly. Endocrine. 2015;48:653-660.

10. Vilar L, Vilar CF, Lyra R, et al. Acromegaly: clinical features at diagnosis. Pituitary. 2017;20:22-32.

11. Lang RM, Badano LP, Mor-Avi V, et al. Recommendations for cardiac chamber quantification by echocardiography in adults: an update from the American Society of Echocardiography and the European Association of Cardiovascular Imaging. Eur Heart J Cardiovasc Imaging. 2015;16:233-270.

12. Frara S, Maffezzoni F, Mazziotti G, et al. The modern criteria for medical management of acromegaly. Prog Mol Biol Transl Sci. 2016;138:63-83.

13. Dineen R, Stewart PM, Sherlock M. Acromegaly. QJM. 2017;110:411-420.
14. dos Santos Silva CM, Gottlieb I, Volschan I, et al. Low frequency of cardiomyopathy using cardiac magnetic resonance imaging in an acromegaly contemporary cohort. J Clin Endocrinol Metab. 2015;100:4447-4455.

15. Kleijn SA, Brouwer WP, Aly MF, et al. Comparison between three-dimensional speckle-tracking echocardiography and cardiac magnetic resonance imaging for quantification of left ventricular volumes and function. Eur Heart J Cardiovasc Imaging. 2012;13:834-839.

16. Hoit BD. Left atrial size and function: role in prognosis. J Am Coll Cardiol. 2014;63:493-505.

17. Myers JA, Lunn KF, Bright JM. Echocardiographic findings in 11 cats with acromegaly. J Vet Intern Med. 2014;28:1235-1238.

18. Aono J, Nobuoka S, Nagashima J, et al. Heart failure in 3 patients with acromegaly: echocardiographic assessment. Intern Med. 1998;37:599-603.

19. Colao A, Marzullo P, Di Somma C, et al. Growth hormone and the heart. Clin Endocrinol (Oxf). 2001;54:137-154.

20. Bogazzi F, Lombardi M, Strata E, et al. High prevalence of cardiac hypertophy without detectable signs of fibrosis in patients with untreated active acromegaly: an in vivo study using magnetic resonance imaging. Clin Endocrinol (Oxf). 2008;68:361-368.

21. Sacca L, Napoli R, Cittadini A. Growth hormone, acromegaly, and heart failure: an intricate triangulation. Clin Endocrinol (Oxf). 2003;59:660-671.

22. Powlson AS, Gurnell M. Cardiovascular disease and sleep-disordered breathing in acromegaly. Neuroendocrinology. 2016;103:75-85.

23. Hayashi S, Yamada H, Bando M, et al. Optimal analysis of left atrial strain by speckle tracking echocardiography: P-wave versus R-wave trigger. Echocardiography. 2015;32:1241-1249.

How to cite this article: Kormányos Á, Domsik P, Kalapos A, et al. Three-dimensional speckle tracking echocardiographyderived left atrial deformation analysis in acromegaly (Results from the MAGYAR-Path Study). Echocardiography. 2018;35:975-984. https://doi.org/10.1111/echo.13860 